\title{
Model Independent Constraints on Non-electronic Flavors in the Solar Boron Neutrino Flux
}

\author{
S. Dev, Sanjeev Kumar ${ }^{\dagger}$ and Surender Verma \\ Department of Physics, Himachal Pradesh University, \\ Shimla, India-171005.
}

\begin{abstract}
We perform the most general model independent analysis of the latest 391-Day Salt Phase SNO Data Set incorporating the Super-Kamiokande ES flux measurement and obtain bounds on the antineutrino and sterile neutrino flux in the solar ${ }^{8} \mathrm{~B}$ neutrino flux reaching the detectors on the earth. The muon/ tauon antineutrino flux is found to be disallowed at $1.4 \sigma$ C.L. The sterile flux is found to be non-zero at about 1.26 standard deviations.
\end{abstract}

The electron neutrinos coming from the Sun were first detected through their Charge Current (CC) interactions with Chlorine 1. The CC reactions detect electron neutrinos only since they are insensitive to the neutrinos of other flavors. The $\nu_{e}$ fluxes measured in different solar neutrino experiments [1, 2] were found to be smaller than the corresponding Standard Solar Model (SSM) 3] estimates giving rise to the solar neutrino problem (SNP). The measurement of solar ${ }^{8} \mathrm{~B}$ neutrino flux through the elastic scattering (ES) reaction at Super-Kamiokande [4] and through the neutral current (NC) reaction at SNO [5] were two major steps towards the resolution of the SNP. The ES flux measurement of SNO agrees with the ES flux measurement at Super-Kamiokande even though the latter have lesser precision. The ES flux at Super-Kamiokande was found to be larger than the SNO CC flux. Interpreting this excess as the contribution of non- electronic neutrino flux resulting from the flavor conversion of electron neutrinos, the hypothesis of the oscillation of solar ${ }^{8} \mathrm{~B}$ neutrinos was, indirectly, established at 3.2 $\sigma$ C.L. 4. A more direct evidence for the flavor conversion of solar ${ }^{8} \mathrm{~B}$ neutrinos was provided by the NC flux measurement of the boron neutrino flux at SNO [5] which was consistent with the SSM prediction for the solar ${ }^{8} \mathrm{~B}$ neutrino flux. This was followed by the independent observation of the oscillations of the terrestrial antineutrinos at KamLAND [6] with the LMA parameters. After these pioneering experiments, neutrino masses and, consequent, oscillations have been established as a physical reality. After the observation of neutrino masses through oscillations, neutrino magnetic moments are an inevitable consequence in the standard model (SM) and beyond.

${ }^{*} \mathrm{E}-\mathrm{mail}: \operatorname{dev} 5703 @ y a h o o . c o m$

${ }^{\dagger} \mathrm{E}$-mail : sanjeev3kumar@yahoo.co.in 
The KamLAND results, directly, ruled out many other possible solutions of the SNP including the resonant spin flavor precession (RSFP) solution resulting from the interaction of a non-vanishing neutrino magnetic moment with the solar magnetic fields. However, the presently available solar neutrino data do not rule out the subdominant contributions of the spin flavor precession (SFP) accompanying the dominant LMA flavor conversion. The SFP is very small for the neutrino parameters in the LMA region but the SFP conversion could be, significantly, enhanced under suitable conditions [7]. A particularly interesting scenario 8] has, recently, been discussed in the literature with non-vanishing transition magnetic moment in the $\mu-\tau$ sector. In this scenario, the final solar neutrino flux can be a mixture of $\nu_{e}, \nu_{\mu}, \nu_{\tau}, \bar{\nu}_{\mu}, \bar{\nu}_{\tau}$ and there will be a direct correlation between the relative fluxes of different neutrino families and the solar magnetic fields. Therefore, it is of considerable importance to constrain the probable non-electronic solar antineutrino flux in a model independent manner keeping, also, in mind the, rather, stringent constraints on the solar electron antineutrino flux 9] since the possible presence of these effects, even at the subdominant level, may affect our present knowledge of the neutrino masses and mixings. Consequently, it is of utmost importance to constrain the possible presence of these subdominant effects from the analysis of solar and atmospheric neutrino data. In the present work, we intend to constrain the possible presence of subdominant SFP transitions and transitions into sterile neutrinos in addition to the dominant LMA transitions in a model independent manner so that the constraints derived in the present work not only apply to the usual SFP transitions [10] but, also, to the more exotic SFP scenarios studied in the recent past [7, 8].

The possibility of transitions into active antineutrinos and sterile neutrinos accompanying the dominant LMA transitions has been examined by several authors [11, 12] in the recent past through a model independent analysis of the Super-Kamiokande and SNO data. In the present work, we reexamine this possibility in the light of the latest 391-Day Salt Phase SNO Data Set and the Super-Kamiokande measurement of the ${ }^{8} \mathrm{~B}$ flux through the ES reaction.

If the solar ${ }^{8} \mathrm{~B}$ neutrinos undergo flavor conversion into active neutrinos only and there are no transitions into antineutrinos, the $\mathrm{CC}$, NC and ES fluxes are no longer independent. The ES flux can be obtained from the $\mathrm{CC}$ and NC fluxes by using the relation [13]

$$
\phi_{E S}=r \phi_{N C}+(1-r) \phi_{C C}
$$

where ' $\mathrm{r}$ ' is the ratio of the ES cross-section of the $\nu_{\mu} / \nu_{\tau}$ component to that of $\nu_{e}$. This relation should be satisfied by the global rates as well as in the individual energy bins. Moreover, Eqn. (1) gives rise to correlations between the CC, NC and ES fluxes which should, also, be visible in the SNO data. However, these correlations will be modified in case there are transitions into antineutrinos at the subdominant level. Therefore, the correlations between different SNO fluxes should serve as the test of the LMA MSW solution and may, also, indicate the presence of subdominant transitions.

In this work, we make use of the 391-Day Salt Phase SNO Data Set with enhanced sensitivity. The shape constrained SNO CC, NC and ES fluxes are given by [14]

$$
\begin{aligned}
& \phi_{C C}^{S N O}=(1.72 \pm 0.12) \times 10^{6} \mathrm{~cm}^{-2} s^{-1} \\
& \phi_{N C}^{S N O}=(4.81 \pm 0.34) \times 10^{6} \mathrm{~cm}^{-2} s^{-1}
\end{aligned}
$$




$$
\phi_{E S}^{S N O}=(2.34 \pm 0.27) \times 10^{6} \mathrm{~cm}^{-2} \mathrm{~s}^{-1} .
$$

The correlation coefficients are given by 14

$$
\begin{gathered}
\rho(C C, N C)=-0.400, \\
\rho(C C, E S)=-0.168, \\
\rho(E S, N C)=-0.073,
\end{gathered}
$$

and will be used in the error propagation calculations.

The starting point of our analysis are the relations between the $\mathrm{CC}, \mathrm{NC}$ and $\mathrm{ES}$ fluxes which are measured experimentally and the neutrino/ antineutrino fluxes of various species which may be present in the solar ${ }^{8} \mathrm{~B}$ neutrino flux. We do not parametrize the contribution of the various fluxes in terms of some trigonometric ratios representing the fractions of different neutrino fluxes as is done in Ref. [11] and Ref. [12]. Our approach, although equivalent, is much simpler and more transparent.

The CC, NC and ES fluxes are related to the various neutrino fluxes in a most general way through the relations

$$
\begin{gathered}
\phi_{C C}=\phi_{\nu_{e}}, \\
\phi_{N C}=\phi_{\nu_{e}}+\phi_{\nu_{x}}+\bar{r}_{d} \phi_{\bar{\nu}_{e}}+\bar{r}_{d} \phi_{\bar{\nu}_{x}}, \\
\phi_{E S}=\phi_{\nu_{e}}+r \phi_{\nu_{x}}+\bar{r}_{e} \phi_{\bar{\nu}_{e}}+\bar{r}_{x} \phi_{\bar{\nu}_{x}} .
\end{gathered}
$$

Here, $\phi_{\nu_{e}}$ is the flux of $\nu_{e}, \phi_{\nu_{x}}$ is the flux of $\nu_{\mu} / \nu_{\tau}, \phi_{\bar{\nu}_{e}}$ is the flux of $\bar{\nu}_{e}$ and $\phi_{\bar{\nu}_{x}}$ is the flux of $\bar{\nu}_{\mu} / \bar{\nu}_{\tau}$. We have assumed that all these neutrinos come with standard boron neutrino spectrum. The various cross-section ratios are given by

$$
\begin{gathered}
r=\frac{\iiint d E_{\nu} d T d T^{\prime} \phi_{B}\left(E_{\nu}\right) \frac{d \sigma\left(\nu_{x} e \rightarrow \nu_{x} e\right)}{d T}\left(T, E_{\nu}\right) R\left(T, T^{\prime}\right)}{\iiint d E_{\nu} d T d T^{\prime} \phi_{B}\left(E_{\nu}\right) \frac{d \sigma\left(\nu_{e} e \rightarrow \nu_{e} e\right)}{d T}\left(T, E_{\nu}\right) R\left(T, T^{\prime}\right)} \\
\bar{r}_{e}=\frac{\iiint d E_{\nu} d T d T^{\prime} \phi_{B}\left(E_{\nu}\right) \frac{d \sigma\left(\bar{\nu}_{e} e \rightarrow \bar{\nu}_{e} e\right)}{d T}\left(T, E_{\nu}\right) R\left(T, T^{\prime}\right)}{\iiint d E_{\nu} d T d T^{\prime} \phi_{B}\left(E_{\nu}\right) \frac{d \sigma\left(\nu_{e} e \rightarrow \nu_{e} e\right)}{d T}\left(T, E_{\nu}\right) R\left(T, T^{\prime}\right)} \\
\bar{r}_{x}=\frac{\iiint d E_{\nu} d T d T^{\prime} \phi_{B}\left(E_{\nu}\right) \frac{d \sigma\left(\bar{\nu}_{x} e \rightarrow \bar{\nu}_{x} e\right)}{d T}\left(T, E_{\nu}\right) R\left(T, T^{\prime}\right)}{\iiint d E_{\nu} d T d T^{\prime}\left(E_{\nu}\right) \frac{d \sigma\left(\nu_{e} e \rightarrow \nu_{e} e\right)}{d T}\left(T, E_{\nu}\right) R\left(T, T^{\prime}\right)} \\
\bar{r}_{d}=\frac{\iiint d E_{\nu} d T d T^{\prime} \phi_{B}\left(E_{\nu}\right) \frac{d \sigma\left(\bar{\nu}_{x} d \rightarrow \bar{\nu}_{x} p n\right)}{d T}\left(T, E_{\nu}\right) R\left(T, T^{\prime}\right)}{\iiint d E_{\nu} d T d T^{\prime}\left(E_{\nu}\right) \frac{d \sigma\left(\nu_{e} d \rightarrow \nu_{e} p n\right)}{d T}\left(T, E_{\nu}\right) R\left(T, T^{\prime}\right)} .
\end{gathered}
$$

From these relations, we obtain $r=0.15, \bar{r}_{x}=0.12, \bar{r}_{e}=0.19$ and $\bar{r}_{d}=0.95$.

It can be seen from Eqns. (8)-(10) that

$$
\phi_{E S}=r \phi_{N C}+(1-r) \phi_{C C}+\left(\bar{r}_{e}-r \bar{r}_{d}\right) \phi_{\bar{\nu}_{e}}-\left(r \bar{r}_{d}-\bar{r}_{x}\right) \phi_{\bar{\nu}_{x}}
$$

In the absence of antineutrinos, we obtain

$$
\phi_{E S}^{n o \bar{\nu}}=r \phi_{N C}+(1-r) \phi_{C C}
$$


which is the same as Eqn. (1). The effect of electron antineutrino component in the solar boron neutrino flux is to increase the ES flux from $\phi_{E S}^{n o}{ }^{\bar{\nu}}$ while that of muon antineutrino component is to decrease the ES flux from $\phi_{E S}^{n o} \bar{\nu}$. Since, $\bar{\nu}_{e}$ flux is restricted to very small values by the Super-Kamiokande 9] and KamLAND [15], there will not be any significant increase in the ES rate due to the contribution from the electron antineutrinos. Hence, we neglect the $\bar{\nu}_{e}$ flux in our analysis.

A value of ES flux smaller than $\phi_{E S}^{n o} \bar{\nu}$ (the ES flux in the absence of $\phi_{\bar{\nu}_{e}}$ and $\phi_{\bar{\nu}_{x}}$ ) will be a signature for the muon antineutrino component in the solar ${ }^{8} \mathrm{~B}$ neutrino flux. We calculate $\phi_{E S}^{n o} \bar{\nu}$ and its correlation coefficients with $\mathrm{CC}$ and $\mathrm{NC}$ fluxes from Eqn. (16) using the CC and NC fluxes as given in Eqns. (2) and (3) and the correlation coefficient between SNO $\mathrm{CC}$ and NC flux given by Eqn. (5) and obtain

$$
\phi_{E S}^{n o} \bar{\nu}=(2.18 \pm 0.09) \times 10^{6} \mathrm{~cm}^{-2} \mathrm{~s}^{-1},
$$

and

$$
\begin{gathered}
\rho(C C, E S)=0.91, \\
\rho(E S, N C)=0.10 .
\end{gathered}
$$

Thus, the SNO ES flux should be equal to $(2.18 \pm 0.09) \times 10^{6} \mathrm{~cm}^{-2} \mathrm{~s}^{-1}$ and correlated with the $\mathrm{CC}$ and $\mathrm{NC}$ fluxes with above correlation coefficients in case there are transitions into active flavors only.

In case, we have independent measurements of $\mathrm{CC}$ and $\mathrm{NC}$ fluxes, they will be correlated with the ES flux through the correlation coefficients

$$
\begin{aligned}
& \rho(C C, E S)=\frac{(1-r) \sigma_{C C}}{\sqrt{r^{2} \sigma_{N C}^{2}+(1-r)^{2} \sigma_{C C}^{2}}}, \\
& \rho(E S, N C)=\frac{r \sigma_{N C}}{\sqrt{r^{2} \sigma_{N C}^{2}+(1-r)^{2} \sigma_{C C}^{2}}},
\end{aligned}
$$

if there are transitions into active flavors only and no transitions into antineutrinos. In the third ${ }^{3} \mathrm{He}$-phase of SNO, the $\mathrm{CC}$ and $\mathrm{NC}$ measurements will be nearly independent. Then, the experimentally measured ES fluxes should, not only, be related with the measured CC and NC fluxes according to Eqn. (1), but also, it should be correlated with the CC and NC fluxes with the correlation coefficients given by Eqns. (20) and (21).

The central value of the ES flux is clearly larger than the central value of $\phi_{E S}^{n o} \bar{\nu}$. However, SNO ES flux has large errors and includes $\phi_{E S}^{n o} \bar{\nu}$ within one standard deviation. On the other hand, Super-Kamiokande ES flux has much smaller errors. Since, there is very little difference between the various cross-section ratios for Super-Kamiokande and SNO, we can use the Super-Kamiokande ES flux in place of SNO ES flux and combine it with SNO CC and NC fluxes. We treat the Super-Kamiokande ES flux as uncorrelated with the SNO CC and ES fluxes.

The Super-Kamiokande ES rate [4] is

$$
\phi_{E S}^{S K}=(2.35 \pm 0.08) \times 10^{6} \mathrm{~cm}^{-2} \mathrm{~s}^{-1}
$$


and

$$
\phi_{E S}^{S K}-\phi_{E S}^{n o \bar{\nu}}=(0.17 \pm 0.12) \times 10^{6} \mathrm{~cm}^{-2} s^{-1}
$$

which is non-zero at about 1.4 standard deviations. If there are transitions into $\bar{\nu}_{x}$ in addition to the transitions into $\nu_{x}$ component, the ES flux should be smaller than $\phi_{E S}^{n o} \bar{\nu}$. But, we see that it is actually larger than $\phi_{E S}^{n o} \bar{\nu}$ upto $1.4 \sigma$. Thus, the Super-Kamiokande data excludes any antineutrino production at $1.4 \sigma$ C.L.. However, arbitrary admixtures of $\phi_{\nu_{x}}$ and $\phi_{\bar{\nu}_{x}}$ are allowed above $1.4 \sigma$ C.L..

We eliminate $\phi_{\nu_{e}}$ from Eqns. (9) and (10) using Eqn. (8) to obtain

$$
\begin{gathered}
\phi_{\nu_{x}}+\bar{r}_{d} \phi_{\bar{\nu}_{x}}=\phi_{N C}-\phi_{C C}-\bar{r}_{d} \phi_{\bar{\nu}_{e}}, \\
r \phi_{\nu_{x}}+\bar{r}_{\mu} \phi_{\bar{\nu}_{x}}=\phi_{E S}-\phi_{C C}-\bar{r}_{e} \phi_{\bar{\nu}_{e}} .
\end{gathered}
$$

If the value of $\phi_{\bar{\nu}_{e}}$ is known, the above set of linear equations can be solved for $\phi_{\nu_{x}}$ and $\phi_{\bar{\nu}_{x}}$. The formal solutions of Eqns. (24) and (25) can be written as

$$
\begin{gathered}
\phi_{\bar{\nu}_{x}}=\frac{1}{r \bar{r}_{d}-\bar{r}_{x}}\left[r \phi_{N C}+(1-r) \phi_{C C}-\phi_{E S}+\left(\bar{r}_{e}-r \bar{r}_{d}\right) \phi_{\bar{\nu}_{e}}\right], \\
\phi_{\nu_{x}}=\frac{1}{r \bar{r}_{d}-\bar{r}_{x}}\left[\bar{r}_{d} \phi_{E S}-\bar{r}_{x} \phi_{N C}-\left(\bar{r}_{d}-\bar{r}_{x}\right) \phi_{C C}+\bar{r}_{d}\left(\bar{r}_{e}-\bar{r}_{x}\right) \phi_{\bar{\nu}_{e}}\right] .
\end{gathered}
$$

Since, $\phi_{\bar{\nu}_{e}}$ is restricted to very small values by Super-Kamiokande [9] and KamLAND [15], we neglect the $\phi_{\bar{\nu}_{e}}$ term in the above equations. So,

$$
\begin{gathered}
\phi_{\bar{\nu}_{x}}=\frac{r \phi_{N C}+(1-r) \phi_{C C}-\phi_{E S}}{r \bar{r}_{d}-\bar{r}_{x}}, \\
\phi_{\nu_{x}}=\frac{\bar{r}_{d} \phi_{E S}-\bar{r}_{x} \phi_{N C}-\left(\bar{r}_{d}-\bar{r}_{x}\right) \phi_{C C}}{r \bar{r}_{d}-\bar{r}_{x}} .
\end{gathered}
$$

Since, $\phi_{E S}$ is larger than $\phi_{E S}^{n o} \bar{\nu}=r \phi_{N C}+(1-r) \phi_{C C}$, the central value of muon antineutrino flux will be negative and only upper bounds on it can be obtained. Substituting the SNO fluxes from Eqns. (2)-(4) and using the correlation coefficients from Eqns. (5)-(7), the active non-electronic antineutrino flux is found to be

$$
\phi_{\bar{\nu}_{x}}=(-7.0 \pm 13.5) \times 10^{6} \mathrm{~cm}^{-2} s^{-1} .
$$

The result is consistent with zero within one standard deviation.

If we use the Super-Kamiokande ES flux, given by Eqn. (22) instead of the SNO ES flux we obtain

$$
\phi_{\bar{\nu}_{x}}=(-7.6 \pm 5.3) \times 10^{6} \mathrm{~cm}^{-2} s^{-1}
$$

The upper bound on $\phi_{\bar{\nu}_{x}}$ is

$$
\phi_{\bar{\nu}_{x}}<3.0 \times 10^{6} \mathrm{~cm}^{-2} \mathrm{~s}^{-1} \text { at } 2 \sigma \text { C.L.. }
$$

It is found that the value of $\phi_{\bar{\nu}_{x}}$ becomes negative below $1.4 \sigma$ C.L.. However, the data is not precise enough to restrict the larger values of antineutrino flux. For instance, $\phi_{\bar{\nu}_{x}}=$ $5.69 \times 10^{6} \mathrm{~cm}^{-2} \mathrm{~s}^{-1}$ is allowed at $2.5 \sigma$. 
The total active neutrino flux is

$$
\phi_{\text {active }}=\phi_{\nu_{e}}+\phi_{\nu_{x}}+\phi_{\bar{\nu}_{x}}
$$

Substituting the values of $\phi_{\nu_{e}}, \phi_{\nu_{x}}$ and $\phi_{\bar{\nu}_{x}}$ from Eqns. (8), (29) and (28) in Eqn. (33), we obtain

$$
\phi_{\text {active }}=\frac{1}{r \bar{r}_{d}-\bar{r}_{x}}\left[\left(r-\bar{r}_{x}\right) \phi_{N C}+\left(1-\bar{r}_{d}\right)\left((1-r) \phi_{C C}-\phi_{E S}\right)\right] .
$$

We note that in the limit $\bar{r}_{d} \sim 1, \phi_{E S} \sim r \phi_{N C}+(1-r) \phi_{C C}$ and so $\phi_{\text {active }} \sim \phi_{N C}$. The sterile flux can, thus, be calculated from the relation

$$
\phi_{\text {sterile }}=\phi_{B}^{S S M}-\phi_{\text {active }}
$$

where the SSM prediction for the total boron neutrino flux is [16]

$$
\phi_{B}^{S S M}=(5.69 \pm 0.91) \times 10^{6} \mathrm{~cm}^{-2} \mathrm{~s}^{-1} .
$$

Using the SNO CC and NC fluxes alongwith the Super-Kamiokande ES flux, sterile neutrino flux is found to be

$$
\phi_{\text {sterile }}=1.25 \pm 0.99 \times 10^{6} \mathrm{~cm}^{-2} \mathrm{~s}^{-1},
$$

which is non-zero at about 1.26 standard deviations and gives a $2 \sigma$ C.L. upper bound of

$$
\phi_{\text {sterile }}<3.23 \times 10^{6} \mathrm{~cm}^{-2} \mathrm{~s}^{-1} \text {. }
$$

In order to compare our results with those obtained by Chauhan and Pulido [12, we calculate the ratio of non-electronic neutrino flux to the total active (neutrino+antineutrino) flux:

$$
\sin ^{2} \psi=\frac{\phi_{\nu_{x}}}{\phi_{\nu_{x}}+\phi_{\bar{\nu}_{x}}}
$$

On substituting $\phi_{\nu_{x}}$ and $\phi_{\bar{\nu}_{x}}$ from Eqns. (28) and (29) in the above equation, we obtain

$$
\sin ^{2} \psi=\frac{\bar{r}_{d} \gamma-\bar{r}_{x}}{r-\bar{r}_{x}-\left(1-\bar{r}_{d}\right) \gamma}
$$

where

$$
\gamma=\frac{\phi_{E S}-\phi_{C C}}{\phi_{N C}-\phi_{C C}}
$$

The analysis of Ref. [12] is based upon Eqn. (40). Substituting the SNO CC and NC fluxes and Super-Kamiokande ES flux in Eqn. (41) and taking into account the anticorrelation between the SNO CC and NC fluxes, we obtain

$$
\gamma=0.20 \pm 0.04
$$

Using this value of $\gamma$, we can calculate the value of $\sin ^{2} \psi$ from Eqn. (40) to obtain

$$
\sin ^{2} \psi=3.5 \pm 2.2
$$


which is $1.1 \sigma$ above unity. Hence, no neutrino-antineutrino admixture is allowed upto 1. $1 \sigma$. However, arbitrary neutrino-antineutrino admixtures are allowed in the range 1.1- 1.6 $\sigma$. For example,

$$
\sin ^{2} \psi=0.5 \text { at } 1.36 \sigma \text { C.L. }
$$

Our results differ from those obtained by Chauhan and Pulido [12] because we incorporate the Super-Kamiokande flux in the analysis from the very start instead of putting it as a constraint at the end and, also, because we have taken into account the anticorrelation between the SNO CC and NC fluxes.

Now, we consider a special case in which there are transitions only into active antineutrinos and no transitions into active neutrinos. This will allow us to obtain the maximum possible antineutrino flux allowed by the data. In this scenario, we have

$$
\phi_{\bar{\nu}_{x}}=\frac{\phi_{N C}-\phi_{C C}}{\bar{r}_{d}}
$$

which gives

$$
\phi_{\bar{\nu}_{x}}=(3.26 \pm 0.41) \times 10^{6} \mathrm{~cm}^{-2} s^{-1} .
$$

Thus, the $2 \sigma$ upper bound on the antineutrino flux is

$$
\phi_{\bar{\nu}_{x}}<4.08 \times 10^{6} \mathrm{~cm}^{-2} \mathrm{~s}^{-1}
$$

In conclusion, a non-electronic antineutrino component in the solar boron neutrino flux is ruled out by the latest 391-Day Salt Phase SNO Data Set and the Super-Kamiokande ES measurement at $1.4 \sigma$ C.L.. However, arbitrary $\nu_{x}-\bar{\nu}_{x}$ admixtures are still allowed above this confidence level. The upper bound on muon/tauon antineutrino flux coming from the Sun is $\phi_{\bar{\nu}_{x}}<3.0 \times 10^{6} \mathrm{~cm}^{-2} \mathrm{~s}^{-1}$ at $2 \sigma$ C.L.. With the future precision measurements of the $\mathrm{CC}$ and NC fluxes at SNO and ES flux at Super-Kamiokande, it will be possible to further constrain the $\nu_{x}-\bar{\nu}_{x}$ admixtures present in the solar boron neutrino flux to smaller values. Combining the Super-Kamiokande ES flux with the SNO CC and NC fluxes, the sterile flux is found to be non-zero at 1.26 standard deviations. The upper bound on the sterile flux is $3.23 \times 10^{6} \mathrm{~cm}^{-2} \mathrm{~s}^{-1}$ at $2 \sigma$ C.L.

\section{Acknowledgments}

One of the authors (S. K.) acknowledges the financial support provided by Council for Scientific and Industrial Research (CSIR), Government of India. The research work of the other two authors (S. D. and S. V.) is supported by the Board of Research in Nuclear Sciences (BRNS), Department of Atomic Energy, Government of India vide Grant No. 2004/37/23/BRNS/399. 


\section{References}

[1] R. Davis, Jr., Phys. Rev. Lett. 12, (1968) 303.

[2] GALlEX Collab. (W. Hampel et al.), Phys. Lett. B 447 (1999) 127.

[3] J. N. Bahcall, Nuc. Phys. (Proc. Suppl.), B118, 77 (2003)

[4] Super-Kamiokande Collab. (S. Fukuda et al.), Phys. Lett. B 539, 179 (2002).

[5] SNO Collab. (Q. R. Ahmad et al.), Phys. Rev. Lett. 89, 011301 (2002)

[6] KamLAND Collab. (K. Eguchi et al., ), Phys. Rev. Lett. 90, 021802 (2003).

[7] M. M. Guzzo, P. C. de Holanda, and O. L. G. Peres, Phys. Lett. B591, 1 (2004) and references cited therein.

[8] M. M. Guzzo, P. C. de Holanda and O. L. G. Peres, hep-ph/0504185.

[9] Super-Kamiokande Collab. (D. W. Liu et al.), Phys. Rev. Lett. 93, 021802, 2004.

[10] C. S. Lim and W. J. Marciano, Phys. Rev. D37, 1368 (1988); E. Kh. Akhmedov, Sov. Phy. JETP 68, 690 (1989); B. C. Chauhan, S. Dev and U. C. Pandey, Phys. Rev. D59, 083002 (1999); E. Kh. Akhmedov and J. Pulido, Phy. Lett. B485, 178 (2000); ibid. B529, 193 (2002); ibid. B553, 7 (2003).

[11] S. K. Kang and C. S. Kim, hep-ph/0306210 and hep-ph/0403059.

[12] Bhag C. Chauhan and João Pulido, hep-ph/0406277.

[13] V. D. Barger, D. Marfatia and K. Whinnant, Phys. Rev. Lett. 88, 011302 (2002).

[14] SNO Collab. (B. Aharmin et al.), nucl-ex/0502021.

[15] KamLAND Collab. (K. Eguchi et al.), Phys. Rev. Lett. 92, 071301, 2004.

[16] J. N. Bahcall, A. M. Serenelli, and S. Basu, astro-ph/0412440 